Orthopäde 2016 4 45:687-694

DOI 10.1007/s00132-016-3265-6

Online publiziert: 1. Juni 2016

(c) Springer-Verlag Berlin Heidelberg 2016

CrossMark
T. D. Lerch - S. D. Steppacher - E. F. Liechti - K. A. Siebenrock - M. Tannast

Klinik und Poliklinik für Orthopädische Chirurgie, Inselspital, Universität Bern, Bern, Schweiz

\section{Periazetabuläre Osteotomie nach Ganz}

\author{
Indikationen, Technik und Ergebnisse \\ 30 Jahre nach Erstbeschreibung
}

die Jahrtausendwende hat die Indikationen für die PAO erweitert. Die ausgeprägte azetabuläre Retroversion wurde erkannt als mögliche Ursache für relevante Hüftbeschwerden im Rahmen eines anterioren FAI. Sie ist unbehandelt verbunden mit einem deutlich erhöhten Risiko der Ausbildung einer sekundären Coxarthrose [9]. Bei der azetabulären Retroversion ist die Fläche der Facies lunata praktisch normal, während deren räumliche Orientierung inkorrekt ist [12]. Aufgrund der speziellen Beckenmorphologie ist das Azetabulum außenrotiert [16]. Mit derselben Schnittführung wie bei der klassischen PAO kann das Azetabulum korrekt reorientiert werden.

In dieser Arbeit werden die aktuellsten Informationen hinsichtlich Indikationen, chirurgischer Technik sowie Langzeitresultate der PAO zusammengefasst.

\section{Indikationen}

Für die periazetabuläre Osteotomie sind zwei Indikationen beschrieben: (1) die Hüftdysplasie und (2) die ausgeprägte azetabuläre Retroversion (• Tab. 2; - Abb. 1).
Die Hüftdysplasie ist definiert als eine defizitäre laterale und anteriore azetabuläre Überdachung des Femurkopfes und wird im Wesentlichen gemäss neuesten Studien durch einen lateralen ZentrumEck-Winkel von weniger als $22^{\circ}$ [21] definiert (• Tab. 1; • Abb. 2a; [10]). Weitere diagnostische Parameter sind ein azetabulärer Index $>14^{\circ}$ und eine Femurkopfextrusion > $27 \%$ (• Abb. 2a). Bei der Hüftdysplasie ist die Beckenform häufig rektangulär (• Abb. 2b) und das Hemipelvis innenrotiert (-Abb. 2c; [16]).

Die ausgeprägte azetabuläre Retroversion ist definiert durch drei radiologische Zeichen (•Abb. 2a), welche alle vorhanden sein müssen: das Überkreuzungszeichen [6] der Vorder- und Hinterwand, das positive Spina-ischiadica-Zeichen [5] und ein positives Hinterwandzeichen [17]. Der Retroversionsindex sollte $>30 \%$ sein als Zeichen einer akzentuierten Retroversion (• Abb. 2a). Das Hemipelvis ist im Gegensatz zur Hüftdysplasie eher trapezoid (• Abb. 2b) und das Hemipelvis typischerweise außenrotiert (• Abb. 2c; [16]). und führt zu keiner relevanten Verän rung der Anatomie des Geburtskanales (• Tab. 1).

Die Operationstechnik hat sich auch mehr als 30 Jahre nach der Erstbeschreibung nur unwesentlich verändert. Es wurden zwar verschiedene Variationen der Hautschnittführung, der tiefen Präparation und der Fixation des azetabulären Fragmentes beschrieben, aber die eigentliche Sequenz der Osteotomien hat sich bis heute nicht verändert.

Die Erstbeschreibung des femoroazetabulären Impingements [4] (FAI) um
Tab. 1 Vorteile und Nachteile der periazetabulären Osteotomie [4]

\begin{tabular}{lc}
\hline Vorteile & Nachteile \\
\hline - Ein Zugang & - Anspruchsvolle Opera- \\
- Sehr versatil hinsichtlich der azetabulären Korrekturamplituden & tionstechnik \\
- Geringe Gefährdung der arteriellen Blutzufuhr zum Azetabulum & - Lernkurve \\
- Integrität des Hinterpfeilers (Schutz für N. ischiadicus, schnellere & - Nur bei geschlossener \\
& Mobilisation) \\
- Erhalt des Geburtskanales & \\
- Inhärente Stabilität durch polygonale Schnittführung \\
- Möglichkeit der gleichzeitigen Osteochondroplastie des Femur- \\
kopfes
\end{tabular}


Tab. 2 Indikationen, Kontraindikationen und Hilfestellung zur Diagnosestellung für die klassische und die antevertierende periazetabuläre Osteotomie (PAO). FAl femoroazetabuläres Impingement

\begin{tabular}{|c|c|c|}
\hline Parameter & Hüftdysplasie & FAI bei azetabulärer Retroversion \\
\hline $\begin{array}{l}\text { Radiologische Dia- } \\
\text { gnose [21] }\end{array}$ & $\begin{aligned} \text { - } & \text { Lateraler Zentrum-Eck-Winkel } \\
& <22^{\circ} \\
\text { - } & \text { Azetabulärer Index }>14^{\circ} \\
\text { - } & \text { Femurkopfextrusion }>27 \% \\
\text { - } & \text { Craniocaudale Überdachung } \\
& <69 \% \\
\text { - } & \text { Vordere Überdachung }<14 \% \\
\text { - } & \text { Hintere Überdachung }<35 \%\end{aligned}$ & $\begin{array}{l}\text { - Positives Überkreuzungszeichen } \\
\text { [11] } \\
\text { - Positives Spina-ischiadica-Zei- } \\
\quad \text { chen [7] } \\
\text { - Positives Hinterwandzeichen [23] } \\
\text { - Retroversionsindex > 30\% }\end{array}$ \\
\hline Beckenform & $\begin{array}{l}\text { Typischerweise innenrotiertes } \\
\text { Hemipelvis [22] }\end{array}$ & $\begin{array}{l}\text { Typischerweise außenrotiertes } \\
\text { Hemipelvis [22] }\end{array}$ \\
\hline Pathomechanismus & $\begin{array}{l}\text { Statische Überlastung durch defi- } \\
\text { zitäre Überdachung }\end{array}$ & $\begin{array}{l}\text { Anteriores femoroazetabuläres } \\
\text { Impingement durch zu prominente } \\
\text { azetabuläre Vorderwand }\end{array}$ \\
\hline Indikation & $\begin{array}{l}\text { Radiologische Diagnose einer } \\
\text { Hüftdysplasie mit entsprechender } \\
\text { Klinik }\end{array}$ & $\begin{array}{l}\text { Radiologische Diagnostik einer } \\
\text { ausgeprägten azetabulären Retro- } \\
\text { version mit entsprechender Klinik }\end{array}$ \\
\hline Klinik & $\begin{array}{l}\text { Meist exzessiver Bewegungsum- } \\
\text { fang [20] }\end{array}$ & $\begin{array}{l}\text { Meist eingeschränkter Bewegungs- } \\
\text { umfang [8] }\end{array}$ \\
\hline $\begin{array}{l}\text { Absolute Kontraindi- } \\
\text { kationen }\end{array}$ & $\begin{array}{l}\text { - Arthrose } \geq \text { Grad } 2 \text { nach Tönnis } \\
\text { - Offene Y-Wachstumsfuge } \\
\text { - Hohe Luxation } \\
\text { - Sekundärpfanne } \\
\text { - Verschlechterung der Kongru- } \\
\text { enz in der Abduktionsaufnahme }\end{array}$ & Arthrose $\geq$ Grad 2 nach Tönnis \\
\hline $\begin{array}{l}\text { Relative Kontraindi- } \\
\text { kationen }\end{array}$ & $\begin{array}{l}\text { Alter }>40 \text { Jahre (individueller } \\
\text { Entscheid je nach Knorpelzustand } \\
\text { in präoperativer MRT) }\end{array}$ & $\begin{array}{l}\text { Alter > } 35 \text { Jahre (dann eher Pfan- } \\
\text { nenrandtrimmung) }\end{array}$ \\
\hline Therapie & Klassische PAO [4] & Antevertierende PAO [14] \\
\hline
\end{tabular}

Tab. 3 Übersicht über negative Prädiktoren, welche eine Hüfttotalprothese nach PAO vorhersagen

\begin{tabular}{|c|c|c|}
\hline $\begin{array}{l}\text { Prädiktoren für eine Hüfttotalprothe- } \\
\text { se }\end{array}$ & Risikoquantifizierung & Referenz \\
\hline Alter & $8 \%$ Risikozunahme pro Jahr & [18] \\
\hline Präoperativer Merle-d'Aubigné-Score & $31 \%$ Risikozunahme pro Punkt tiefer & [18] \\
\hline Präoperativer Arthrosegrad nach Tönnis & 3,4-fach erhöhtes Risiko nach 20 Jahren & [18] \\
\hline Hinken präoperativ & 2,8-fach erhöhtes Risiko nach 20 Jahren & [18] \\
\hline $\begin{array}{l}\text { Positiver anteriorer Impingementtest } \\
\text { präoperativ }\end{array}$ & 6,2-fach erhöhtes Risiko nach 20 Jahren & [18] \\
\hline Postoperativer Extrusionsindex & $\begin{array}{l}11 \% \text { Risikozunahme pro Prozent weni- } \\
\text { ger }\end{array}$ & [18] \\
\hline $\begin{array}{l}\text { Lateraler Zentrum-Eck-Winkel unter } 0^{\circ} \\
\text { präoperativ }\end{array}$ & 4,7-fach erhöhtes Risiko nach 9 Jahren & {$[25]$} \\
\hline $\begin{array}{l}\text { Exzessive laterale und proximale Disloka- } \\
\text { tion des Femurkopfes präoperativ }\end{array}$ & 4-fach erhöhtes Risiko nach 9 Jahren & {$[25]$} \\
\hline $\begin{array}{l}\text { Postoperative Breite der Sklerosezone } \\
<2,5 \mathrm{~cm}\end{array}$ & 6-fach erhöhtes Risiko nach 9 Jahren & {$[25]$} \\
\hline Vorhandenes Os acetabuli & 3,6-fach erhöhtes Risiko nach 9 Jahren & {$[25]$} \\
\hline Azetabuläre Anteversion unter $10^{\circ}$ & 4,3-fach erhöhtes Risiko nach 9 Jahren & {$[25]$} \\
\hline
\end{tabular}

\section{Operationstechnik}

\section{Zugang}

Der Patient wird in Rückenlage gelagert und das betroffene Bein steril abgedeckt. Wir verwenden, wenn möglich, eine verkürzte ilioinguinale Inzision (• Abb. 1a), welche etwa $5 \mathrm{~cm}$ dorsal der Spina iliaca anterior superior beginnt und $5 \mathrm{~cm}$ medial davon endet. Die geschwungene Inzision verläuft $2 \mathrm{~cm}$ distal zur Crista iliaca. Diese Inzision führt zu kosmetisch besseren Narben im Vergleich zur ursprünglich beschriebenen SmithPetersen-Inzision, welche aber bei korpulenteren Patienten bei uns immer noch zur Anwendung kommt [3]. Nach Spalten der Subkutis erfolgt die tiefe Präparation im intermuskulären und -nervalen Intervall zwischen dem M. sartorius medial und dem $M$. tensor fasciae latae lateral (• Abb. 1b). Dadurch kommt der direkte Ursprung des M. rectus femoris an der Spina iliaca anterior inferior zum Vorschein, welcher im Unterschied zur Originalbeschreibung heutzutage erhalten wird. Der Ursprung des M. sartorius wird zusammen mit dem Leistenband scharf von der Spina iliaca anterior superior abgelöst. Die Abdominalmuskulatur und der M. iliacus wird subperiostal vom Beckenkamm und der Fossa iliaca mit flektiertem Bein abgelöst, wodurch die Linea terminalis sichtbar wird (- Abb. 1b). Der M. obturatorius internus wird stumpf von der quadrilateralen Fläche mobilisiert und ein Haken auf die Spina ischiadica eingesetzt. Ein zweiter Haken wird auf dem oberen Schambeinast platziert. Das Intervall zwischen dem M. rectus femoris und dem bei der Hüftdysplasie typischerweise hypertrophen M. iliocapsularis wird anschließend dargestellt und mittels Schere stumpf eröffnet, wobei die Gelenkskapsel intakt bleibt. Mit einer gebogenen Schere wird das Os ischium gerade caudal des posteroinferioren Azetabulumhorns palpiert (- Abb. 1b).

\section{Sequenz der Osteotomien}

Unabhängig von der Indikation ist die Sequenz der Osteotomien identisch. Die Mobilisation des Azetabulums vom 
Beckenring erfolgt mittels vier Osteotomien und einer kontrollierten Fraktur (- Abb. 1c).

Die erste Osteotomie ist die inkomplette Ischiumosteotomie, wobei die Incisura ischiadica minor intakt bleiben sollte. Die Osteotomie wird mit einem gebogenen Spezialmeißel im Intervall zwischen der Gelenkskapsel lateral und dem M. iliopsoas medial auf eine Länge von 4-5 cm durchgeführt. Zum Schutz des $\mathrm{N}$. ischiadicus wird die Hüfte extendiert und abduziert.

Die zweite Osteotomie erfolgt am oberen Schambeinast medial der Eminentia iliopectinea. Unter Schutz mit zwei stumpfen Hohmann-Haken wird der obere Schambeinast mit einem LexerMeißel osteotomiert, wobei ein kleiner Knochenkeil mit einer Basis von etwa $2 \mathrm{~mm}$ entfernt wird, was die spätere Mobilisation des azetabulären Fragmentes erleichtert.

Die dritte, supraazetabuläre Osteotomie erfolgt mit einer oszillierenden Säge gerade distal der Spina iliaca anterior superior, was die Durchblutung des azetabulären Fragmentes erhält. Die Abduktorenmuskulatur wird von der $\mathrm{Au}$ Benseite des Beckenkammes mit einem Haken tunneliert und geschützt. Diese Osteotomie reicht senkrecht etwa $4 \mathrm{~cm}$ nach dorsal und endet etwa $2 \mathrm{~cm}$ vor der Linea terminalis ( $\bullet$ Abb. 1c).

Die vierte retroazetabuläre Osteotomie wird zuerst mittels Meißel angezeichnet, ist zur Dritten etwa $110-120^{\circ}$ anguliert und verläuft parallel zum Hinterpfeiler, wobei eine intakte Knochenbrücke von etwa $1 \mathrm{~cm}$ erhalten bleiben sollte (- Abb. 1c). Mittels Meißel werden die ersten 15-20 mm von der Beckeninnenseite osteotomiert.

Die kontrollierte Fraktur, welche die erste und vierte Osteotomie verbindet, erfolgt mittels Mobilisation des azetabulären Fragmentes durch eine 4,5-mmSchanzschraube durch die Spina iliaca anterior inferior, einen breiten LexerMeißel in der dritten Osteotomie und eines oder zwei AO-Spreizer(n) in der vierten Osteotomie (- Abb. 1c). Es ist wichtig, dass das azetabuläre Fragment komplett mobilisiert wird, um eine spätere, ungewollte Lateralisierung zu vermeiden. Die Fraktur kann auch mit einem ent-

Orthopäde 2016 · 45:687-694 DOI 10.1007/s00132-016-3265-6

(c) Springer-Verlag Berlin Heidelberg 2016

T. D. Lerch · S. D. Steppacher - E. F. Liechti · K. A. Siebenrock · M. Tannast Periazetabuläre Osteotomie nach Ganz. Indikationen, Technik und
Ergebnisse 30 Jahre nach Erstbeschreibung

\section{Zusammenfassung}

Die Berner periazetabuläre Osteotomie (PAO) nach Ganz ist eine Operationstechnik zur Behandlung der (1) Hüftdysplasie und des (2) femoroazetabulären Impingements bei einer ausgeprägten azetabulären Retroversion mit dem Ziel, eine sekundäre Coxarthrose zu verhindern oder zumindest herauszuzögern. Im Unterschied zu anderen Beckenosteotomien bleibt der hintere Pfeiler intakt, was die primäre Stabilität der Reorientierung erhöht und den Geburtskanal nicht verändert. Über eine geschwungene verkürzte ilioinguinale Inzision werden vier Osteotomien und eine kontrollierte Fraktur durchgeführt, was eine Reorientierung des Azetabulums mit großem Freiheitsgrad erlaubt. Während die Sequenz der Osteotomien für beide Indikationen identisch ist, unterscheidet sich aber die Reorientierung des Azetabulums. Der Zugang gestattet zudem eine anteriore Kapsulotomie mit femoraler Offset-Korrektur. Obwohl die Operation technisch anspruchsvoll ist, ist die Komplikationsrate verhältnismäßig klein. Der Schlüssel für ein erfolgreiches Langzeitergebnis liegt in der optimalen Reorientierung des Azetabulums bei beiden Indikationen. Klinisch kann mit einer guten azetabulären Einstellung in 80-90\% der Fälle ein gutes bis sehr gutes Resultat nach 10 Jahren erzielt werden. Die kumulative Überlebensrate der weltweit ersten Hüften 20 Jahre nach PAO liegt bei $60 \%$. Die vorläufige Evaluation derselben Serie nach 30 Jahren zeigt eine Überlebensrate von etwa $30 \%$. Die PAO hat sich zu einer standardisierten Therapie der Hüftdysplasie bei Adoleszenten und Erwachsenen etabliert.

\section{Schlüsselwörter}

Adoleszenten · Azetabulum · Coxa · Hüftdysplasie · Femoroazetabuläres Impingement

\section{Bernese periacetabular osteotomy.. Indications, technique and results $\mathbf{3 0}$ years after the first description}

\section{Abstract}

The Bernese periacetabular osteotomy (PAO) is a surgical technique for the treatment of (1) hip dysplasia and (2) femoroacetabular impingement due to acetabular retroversion. The aim of the surgery is to prevent secondary osteoarthritis by improvement of the hip biomechanics. In contrast to other pelvic osteotomies, the posterior column remains intact with this technique. This improves the inherent stability of the acetabular fragment and thereby facilitates postoperative rehabilitation. The birth canal remains unchanged. Through a shortened ilioinguinal incision, four osteotomies and one controlled fracture around the acetabulum are performed. The direction of acetabular reorientation differs for both indications while the sequence of the osteotomies remains the same. This surgical approach allows for a concomitant osteochondroplasty in the case of an aspherical femoral head-neck junction. The complication rate is relatively low despite the complexity of the procedure. The key point for a successful long term outcome is an optimal reorientation of the acetabulum for both indications. With an optimal reorientation and a spherical femoral head, the cumulative survivorship of the hip after 10 years is $80-90 \%$. For the very first 75 patients, the cumulative 20 year survivorship was $60 \%$. The preliminary evaluation of the same series at a 30-year follow-up still showed a survivorship of approximately $30 \%$. The PAO has become the standard procedure for the surgical therapy of hip dysplasia in adolescents and adults.

\section{Keywords}

Acetabulum · Adolescents · Coxa · Hip dysplasia $\cdot$ Femoroacetabular impingement 
a

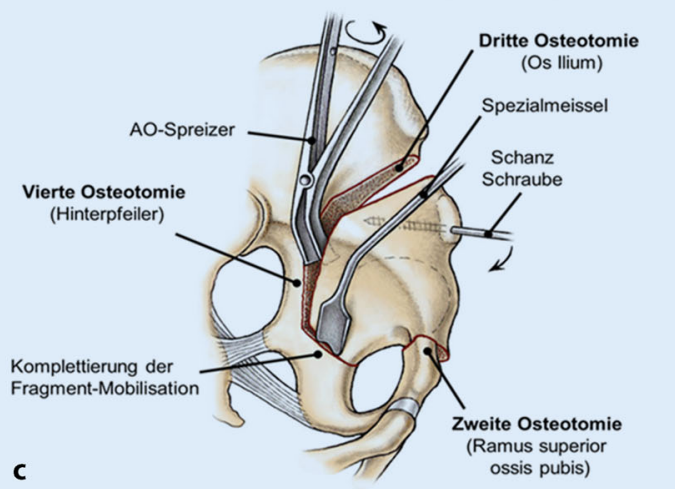

b
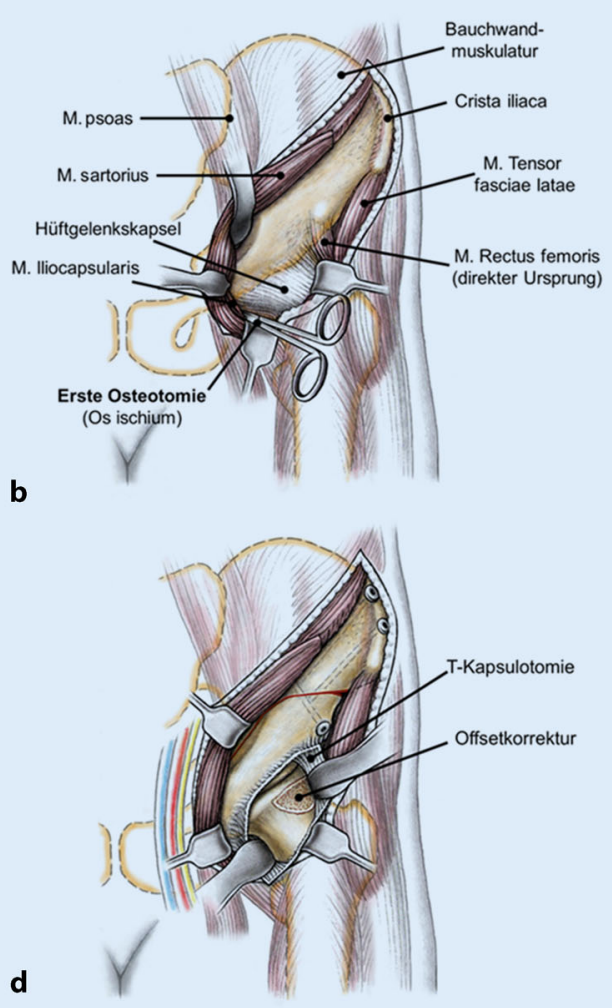

Abb. 1 A Der aktuelle chirurgische Zugang für die periazetabuläre Osteotomie in Bern sowie die Sequenz der Osteotomien sind dargestellt. Die Hautinzision a verläuft ähnlich einer verkürzten ilioinguinalen Inzision geschwungen circa $2 \mathrm{~cm}$ unter dem Beckenkamm. Die tiefe Präparation b verläuft zwischen M. sartorius und M. tensor fasciae latae. Die erste Osteotomie (inkomplette Ischiumosteotomie) erfolgt von ventral im Intervall zwischen der Gelenkskapsel lateral und dem M. iliocapsularis medial.Dieweiteren Osteotomien cerfolgen am oberen Schambeinast, supraazetabulärund entlang des Hinterpfeilers. Durch eine zusätzliche T-förmige Kapsulotomie d kann zudem eine Osteochondroplastie eines allfälligen asphärischen Femurkopfes durchgeführt werden. (Mit freundl. Genehmigung der Autoren)

sprechenden Spezialmeißel komplettiert werden (• Abb. 1c).

\section{Reorientierung des azetabulären Fragmentes}

Die azetabuläre Reorientierung ist der Schlüsselpunkt der gesamten Operation und direkt mit dem Langzeitresultat nach PAO verbunden. Die Korrekturrichtung des azetabulären Fragmentes ist unterschiedlich bei der klassischen PAO im Vergleich zur antevertierenden PAO. Bei der klassischen PAO besteht die Hauptkorrektur in der Regel aus einer Rotation des Fragmentes nach ventral und kaudal, um damit eine ausreichende laterale Überdachung zu erreichen ( $\bullet$ Abb. 2e). Bei der antevertierenden PAO besteht die Hauptkorrektur im Wesentlichen durch eine Innenrotation des Azetabulums (• Abb. 2e), welches aufgrund der speziellen Form des Hemipelvis nach außen rotiert ist. Selten ist eine zusätzliche supraazetabuläre Keilresektion notwendig, um eine genügende Anteversion des Azetabulums zu erreichen [7].

Ziel der Reorientierung des azetabulären Fragmentes ist ein lateraler Zentrum-Eck-Winkel von 23-33 [21], ein antevertiertes Azetabulum ohne Überkreuzungszeichen der Vorder- und Hinterwand, ein Femurkopfextrusionsindex von $17-27 \%$ [15], eine Wiederherstellung der Shenton-Linie sowie der Gelenkskongruenz. Nach temporärer Fixation mittels 2,5-mm-Gewindekirschnerdrähten erfolgt die Überprüfung des Bewegungsumfanges der Hüfte und die intraoperative Evaluation der Reorientierung mittels eines anteroposterioren Röntgenbildes des gesamten Beckens.

\section{Fixation des azetabulären Fragmentes}

Die definitive Fixation erfolgt mit zwei bis drei 3,5-mm-Kortikalisschrauben vom Beckenkamm in das azetabuläre Fragment. Zudem wird eine dritte Kortikalisschraube in anteroposteriorer Richtung vom Pfannenfragment ausgehend von der Spina iliaca anterior inferior in Richtung Iliosakralgelenk platziert (• Abb. 2d). Der am azetabulären Fragment durch die Korrektur entstandene überstehende Knochen wird entfernt und als Autograft in den Osteotomiespalt eingefügt.

\section{Femorale Offsetkorrektur}

Falls sich trotz optimaler Reorientierung des Azetabulums keine Innenrotation von $30^{\circ}$ in $90^{\circ}$-Flexion durchführen lässt, 


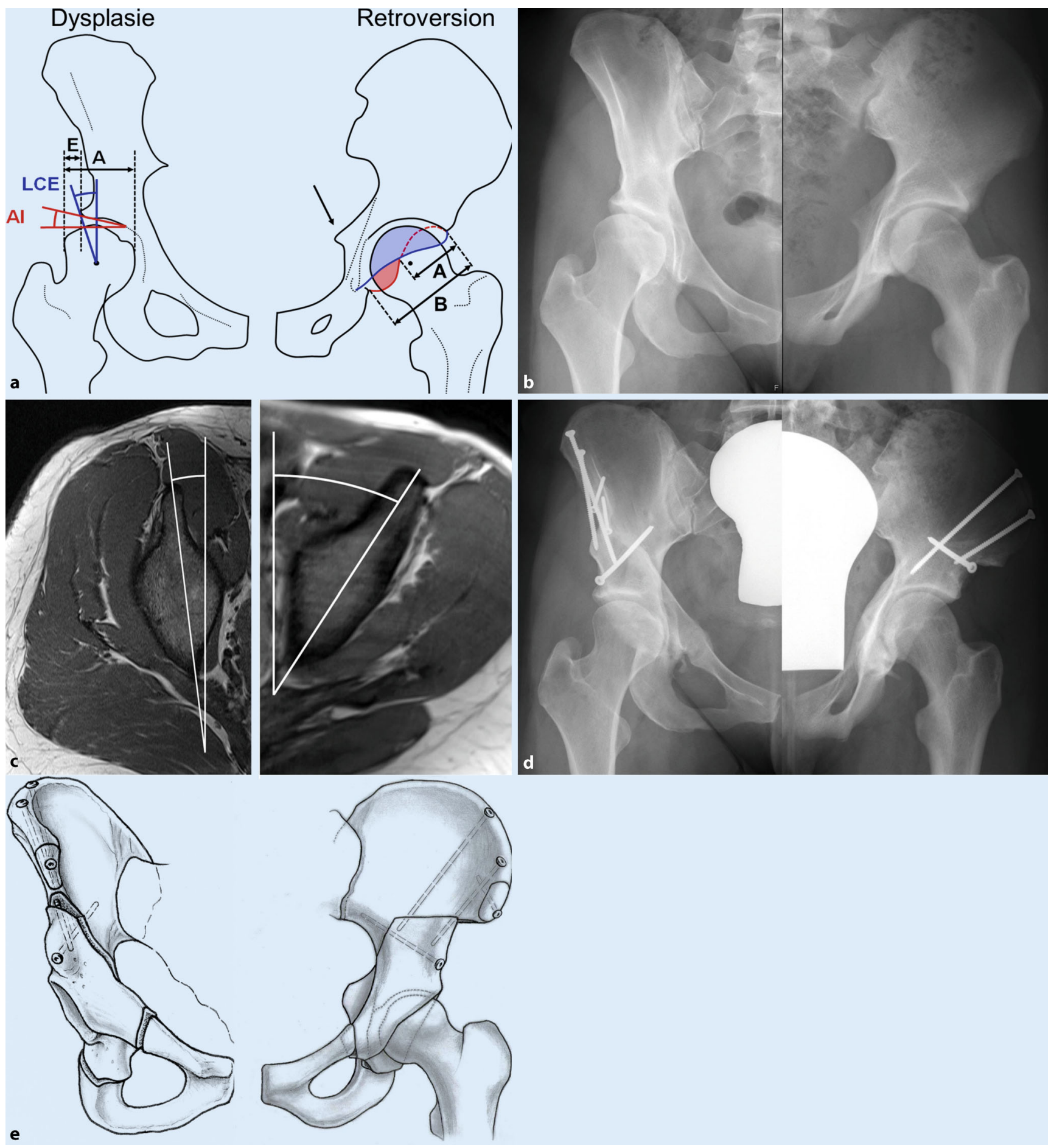

Abb. 2 ム Übersicht über die beiden Indikationen der periazetabulären Osteotomie: klassische Hüftdysplasie (links) und azetabuläre Retroversion (rechts); a Schematische Darstellung einer dysplastischen Hüfte (links) mit Definition des lateralen Zentrum-Eck-Winkels $(L C E)$, des Femurkopfextrusionsindexes $(E / A)$ und des azetabulären Indexes $(A l)$. Rechts schematische Darstellung einer azetabulären Retroversion mit positivem Spina-ischiadica-Zeichen (Pfeil), Überkreuzungszeichen der azetabulären Vorder- und Hinterwand sowie einem positiven Hinterwandzeichen (Punkt); b Konventionell-radiologische Präsentation; c MRT-Schnittbild supraazetabulär, welches eine Innenrotation des Hemipelvis bei der Dysplasie (links) zeigt, während bei der Retroversion das Hemipelvis nach außen rotiert ist (rechts); $\mathbf{d}$ Postoperatives Röntgenbild nach klassischer PAO (links) und antevertierender PAO (rechts); e Schematische Darstellung der azetabulären Korrektur im Rahmen der PAO (Mit freundl. Genehmigung der Autoren) 


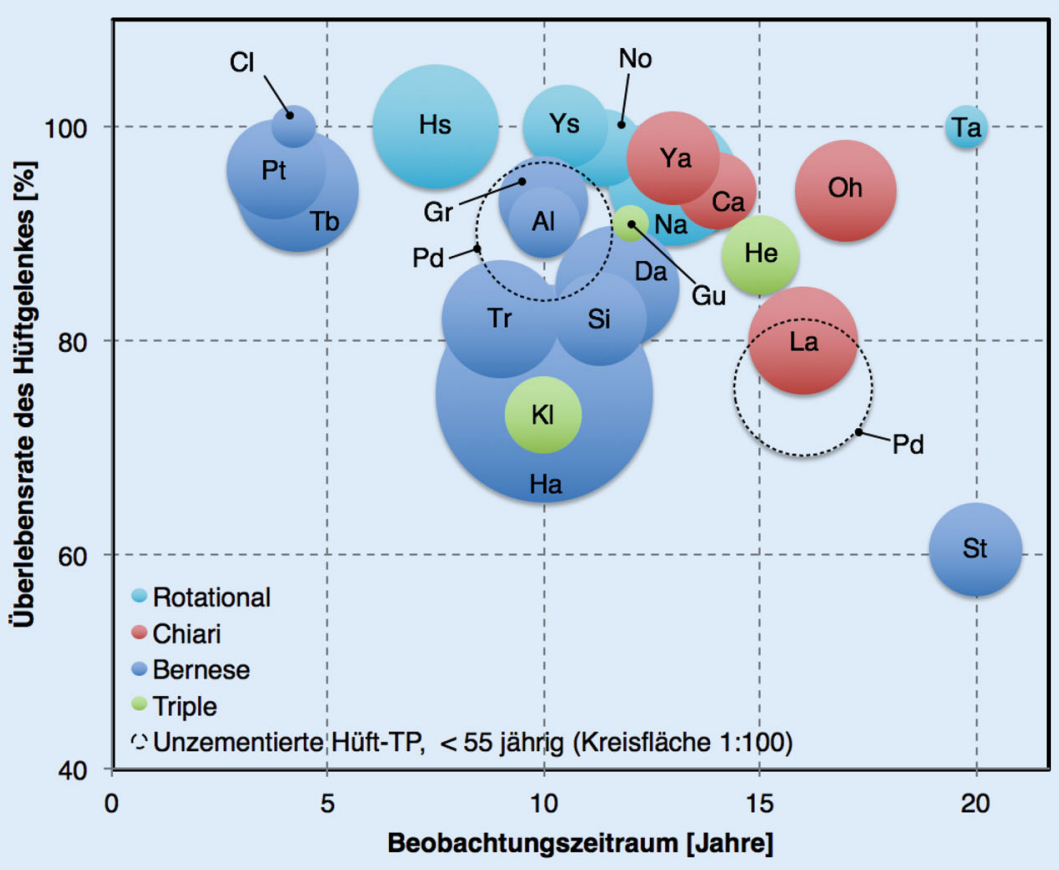

Abb. 3 ॥ Übersicht der Überlebenszeiten des Hüftgelenkes in aktuellen Langzeitstudien verschiedener Umstellungsosteotomien des Azetabulums bei Hüftdysplasie. Der Endpunkt war jeweils eine Konversion zu einer Hüfttotalprothese. Jeder Kreis repräsentiert eine Studie. Die Größe des Kreises ist proportional zur Anzahl Patienten der jeweilgen Studie. Als Vergleichsreferenz sind zusätzlich die Langzeitergebnisse von Primärhüftprothesen bei einem vergleichbaren Patientengut (Alter $<55$ Jahre) eingezeichnet. AlAlbers et al. [1], CaCalvert et al.[27], CIClohisy et al. [28], Da Dahl et al.[29], GrGrammatopoulos et al.[30], Gu Guille et al. [31], Ha Hartig-Andreasen et al. [32], He van Hellemondt et al. [33], Hs Hasegawa et al.[34], KI de Kleuver et al.[35], La Lack et al. [36], Na Nakamura et al. [37], No Nozawa et al. [38], Oh Ohashi et al. [39], Pd Pedersen et al. [40], Pt Peters et al. [41], Si Siebenrock et al. [42], St Steppacher et al. [43], Ta Takatori etal. [44], Tb Trumble et al. [45], Tr Troelsen et al. [46], Ya Yanagimoto et al. [47], Ys Yasunaga et al. [48] (Mit freundl. Genehmigung der Autoren)

muss ein möglicher femoroazetabulärer Konflikt durch eine T-förmige Kapsulotomie evaluiert werden (• Abb. 1d). Bei Dysplasiehüften finden sich häufig asphärische Hüftköpfe [19], welche nach Reorientierung des Azetabulums in kombinierter Flexion und Innenrotation anschlagen können. Mit einem gebogenen Meißel und einer Kugelfräse wird die Konkavität des Schenkelhalses wiederhergestellt.

\section{Wundverschluss}

Die Gelenkskapsel wird mittels resorbierbarer Fäden locker verschlossen. Das Leistenband wird wieder mittels nicht resorbierbarer Nähte an der Spina iliaca anterior superior fixiert und die Abdominalmuskulatur an den Beckenkamm, nach Einlage einer Saugdrainage in die
Fossa iliaca, mit einer fortlaufenden Naht refixiert.

\section{Postoperative Nachbehandlung}

Unmittelbar postoperativ erfolgt die passive Mobilisation mittels einer motorisierten Bewegungsschiene. Das betroffene Bein darf für 8 Wochen mit $15 \mathrm{~kg}$ teilbelastet werden. Die Flexion ist limitiert auf $90^{\circ}$, um einen unnötigen Stress auf das azetabuläre Fragment zu vermeiden. Bei radiologischem Nachweis einer Konsolidation erfolgt nach 2 Monaten der sukzessive Belastungsaufbau.

\section{Ergebnisse}

\section{Klassische PAO bei Hüftdysplasie}

Es gibt gute indirekte Hinweise, dass bei ausgeprägten Hüftdysplasien der
Spontanverlauf der Arthrose zumindest aufgehalten werden kann [13]. Mehrere Langzeitstudien haben die Ergebnisse nach PAO evaluiert (• Abb. 3). Für die erste Serie von 75 Hüften, welche mittels der PAO nach Ganz therapiert wurden, sind die Resultate nach 20 Jahre beschrieben worden [13]. Bei dieser bezüglich vorbestehender Arthrose und Voroperationen sehr heterogenen Gruppe hatten nach 20 Jahren $40 \%$ der Hüften eine Hüfttotalprothese. Dabei waren maßgebend für ein schlechtes Langzeitüberleben der Hüftgelenke folgende Faktoren (- Tab. 3): eine vorbestehende Arthrose, wobei Hüften mit einem Arthrosegrad $>1$ nach Tönnis zu $88 \%$ nach 20 Jahren eine Hüfttotalprothese hatten. Zudem waren ein erhöhtes Alter, vorbestehende starke Schmerzen und Hinken assoziiert mit verfrühtem endoprothetischem Ersatz. Hüften mit einer postoperativ ungenügenden Überdachung (Extrusionsindex $>20 \%$ ) zeigten ebenfalls ein vermindertes Langzeitüberleben. Studien mit der verfeinerten Technik der PAO zeigten einen Erhalt des Hüftgelenkes in 75-93 \% nach 10 Jahren (• Abb. 3). Dabei waren die wichtigsten prädiktiven Faktoren für das Langzeitergebnis die vorbestehende Arthrose und eine optimale azetabuläre Orientierung nach PAO. Dabei muss für ein gutes Langzeitüberleben sowohl eine exzessive azetabuläre Anteversion als auch eine Retroversion des Azetabulums vermieden werden [1]. Zusätzlich ist ein korrektes Kopf-Hals-Offset ausschlaggebend für einen impingementfreien Bewegungsumfang, was reproduzierbar $\mathrm{zu}$ besseren Langzeitergebnissen führt [1].

\section{Antevertierende PAO}

Zwei Studien für die Ergebnisse der antevertierenden PAO bei azetabulärer Retroversion existieren [7, 9]. Dabei wurde in beiden Studien kein endoprothetischer Ersatz der Hüftgelenke nach 2,5 respektive 11 Jahren durchgeführt. Hüftgelenke mit korrektem Kopf-Hals-Offset und azetabulärer Reorientierung (Vermeidung exzessiver azetabulärer Anteversion) hatten weniger Schmerzen und Arthroseprogression nach 11 Jahren [7]. Interne, bisher nicht publizierte Daten 
Tab. 4 Komplikationen der PAO graduiert nach Sink [16]

\begin{tabular}{|c|c|c|c|}
\hline Gradierung & Komplikation & $\begin{array}{l}\text { Prävale } \\
\text { (\%) }\end{array}$ & Literatur \\
\hline \multirow{4}{*}{$\begin{array}{l}1 \text { Komplikation ohne } \\
\text { klinische Relevanz }\end{array}$} & Harnwegsinfektion & $1,5-13$ & {$[2,9]$} \\
\hline & Oberflächlicher Wundinfekt & 5 & [24] \\
\hline & $\begin{array}{l}\text { Heterotope Ossifikationen Grad 1-2 nach } \\
\text { Brooker }\end{array}$ & $1,8-15$ & {$[2,6]$} \\
\hline & Postoperatives Fieber & 18 & [2] \\
\hline \multirow{10}{*}{$\begin{array}{l}2 \text { Komplikation mit } \\
\text { Abweichung vom } \\
\text { normalen Verlauf, aber } \\
\text { ambulante oder } \\
\text { medikamentöse } \\
\text { Behandlung }\end{array}$} & Parese N. cutaneus femoris lateralis & 30 & [2] \\
\hline & Parese N. femoralis & $1,6-5,6$ & {$[13,26]$} \\
\hline & Parese N. ischiadicus & $1,5-7$ & {$[9,13]$} \\
\hline & $\begin{array}{l}\text { Massiver Blutverlust (> } 5 \text { Blutkonserven ver- } \\
\text { abreicht) }\end{array}$ & $3-8$ & {$[2,9]$} \\
\hline & Avulsion Spina iliaca anterior superior & Bis 23 & [3] \\
\hline & Tiefer Wundinfekt & $3-15$ & {$[2,9]$} \\
\hline & Diskontinuität des Hinterpfeilers & $1,5-8,5$ & {$[2,9]$} \\
\hline & Ischiumfraktur & 4,3 & [13] \\
\hline & Stressfraktur Ramus inferior Os pubis & 5 & [24] \\
\hline & $\begin{array}{l}\text { Verzögerte Heilung der Osteotomien } \\
\text { Pseudarthrose am oberen Schambeinast, } \\
\text { nicht revisionsbedürftig }\end{array}$ & $\begin{array}{l}2,6-5 \\
6-16\end{array}$ & $\begin{array}{l}{[4,9]} \\
{[9,24]}\end{array}$ \\
\hline \multirow{6}{*}{$\begin{array}{l}3 \text { Komplikation mit } \\
\text { chirurgischer } \\
\text { Behandlung oder } \\
\text { ungeplanter } \\
\text { Hospitalisation }\end{array}$} & Tiefe Beinvenenthrombose, Lungenembolie & 3 & [9] \\
\hline & Migration des azetabulären Fragmentes & $1,3-5,6$ & {$[4,13]$} \\
\hline & $\begin{array}{l}\text { Revisionsbedürftige heterotope Ossifikatio- } \\
\text { nen Grad 3-4 nach Brooker }\end{array}$ & $1-8,5$ & {$[10,13]$} \\
\hline & $\begin{array}{l}\text { Revisionsbedürftige Pseudoarthrose am obe- } \\
\text { ren Schambeinast }\end{array}$ & 1 & [24] \\
\hline & Revisionsbedürftiges Hämatom & 5 & [2] \\
\hline & Intraartikuläre Osteotomie oder Fraktur & $1-15,5$ & {$[10,13]$} \\
\hline $\begin{array}{l}4 \text { Hüfttotalprothese } \\
\text { oder lebensbedrohliche } \\
\text { Komplikation }\end{array}$ & Nekrose des azetabulären Fragmentes & 4,2 & [13] \\
\hline
\end{tabular}

unserer Klinik zeigen, dass die antevertierende PAO bei der azetabulären Retroversion der Pfannenrandtrimmung nach einem Beobachtungszeitraum von 5 bis 10 Jahren überlegen ist.

\section{Komplikationen}

Trotz der Komplexität der Operation sind für die PAO nur wenige Komplikationen beschrieben. Eine Gesamtkomplikationsrate lässt sich aufgrund der Heterogenität der Berichte nicht allgemeingültig zusammenfassen. Die am häufigsten zu beobachtende Komplikation ist eine transiente oder permanente Schädigung des N. cutaneus femoris lateralis mit einer Prävalenz von bis $30 \%$ [2]. Eine systematische Auflistung der in der Literatur beschriebenen Komplikationen aufgeteilt nach Schweregraden nach Sink [11] fin- det sich in • Tab. 4. Die häufigste revisionsbedürftige Komplikation war eine intraartikuläre Osteotomie, deren Inzidenz mit zunehmender Erfahrung nachweislich reduziert werden konnte [8].

\section{Fazit für die Praxis}

- Die PAO ist eine kausale Therapie zur Verhinderung der frühen Arthroseentwicklung bei jungen Patienten mit Hüftdysplasie und azetabulärer Retroversion. Sie hat sich seit ihrer Erstbeschreibung als chirurgische Standardoperation zur Behandlung der Hüftdysplasie etabliert.

- Die ersten internen Resultate in unserer Klinik konnten zeigen, dass die PAO der Pfannenrandtrimmung bei ausgeprägter azetabulärer Retroversion überlegen ist. Die korrekte
Indikationsstellung und eine frühe Behandlung von symptomatischen Pathologien korreliert direkt mit dem Langzeitergebnis.

- Die hauptsächliche Schwierigkeit für den Operateur ist die optimale Balance von Unter- und Überkorrektur des azetabulären Fragmentes. Eine gleichzeitige Offsetverbesserung bei eingeschränkter Innenrotation nach azetabulärer Reorientierung verbessert die Langzeitprognose relevant.

\section{Korrespondenzadresse}

\section{Prof. Dr. M. Tannast}

Klinik und Poliklinik für Orthopädische Chirurgie, Inselspital, Universität Bern Freiburgstrasse, 3010 Bern, Schweiz moritz.tannast@insel.ch

\section{Einhaltung ethischer Richtlinien}

Interessenkonflikt. T. D. Lerch, S. D. Steppacher, E. F. Liechti und K. A. Siebenrock geben an, dass kein Interessenkonflikt besteht. M. Tannast erhält finanzielle Unterstützung durch den Schweizerischen Nationalfonds (Antrag Nr. PP00P3_144856).

Dieser Beitrag beinhaltet keine von den Autoren durchgeführten Studien an Menschen oder Tieren.

\section{Literatur}

1. Albers CE, Steppacher SD, Ganz R et al (2013) Impingement adversely affects 10 -year survivorship after periacetabular osteotomy for $\mathrm{DDH}$. Clin Orthop Relat Res 471:1602-1614

2. Biedermann R, Donnan L, Gabriel A et al (2008) Complications and patient satisfaction after periacetabular pelvic osteotomy. Int Orthop 32:611-617

3. Crockarell J Jr, Trousdale RT, Cabanela ME et al (1999) Early experience and results with the periacetabular osteotomy. The Mayo Clinic experience. Clin Orthop Relat Res 363:45-53

4. Ganz R, Klaue K, Vinh T et al (1988) A New periacetabular osteotomy for the treatment of hip dysplasias. Clin Orthop Relat Res 232:26-36

5. Ganz R, Parvizi J, Beck M et al (2003) Femoroacetabular impingement: a cause for osteoarthritis of the hip. Clin Orthop Relat Res 417:112-120

6. Hussell JG, Rodriguez JA, Ganz R (1999) Technical complications of the Bernese periacetabular osteotomy. Clin Orthop Relat Res 363:81-92

7. Kalberer F, Sierra RJ, Madan SS et al (2008) Ischial spine projection into the pelvis: a new sign for acetabular retroversion. Clin Orthop Relat Res 466:677-683

8. Kubiak-Langer M, TannastM, MurphySB etal (2007) Range of motion in anterior femoroacetabular impingement. Clin Orthop Relat Res 458:117-124 
9. Matta JM, Stover MD, Siebenrock K (1999) Periacetabular osteotomy through the Smith-Petersen approach. Clin Orthop Relat Res 363:12-32

10. Murphy SDR (2002) Periacetabular osteotomy: preoperative radiographic predictors of outcome. Clin Orthop Relat Res 405:168-174

11. Reynolds D, Lucas J, Klaue K (1999) Retroversion of the acetabulum. A cause of hip pain. J Bone Joint Surg $\operatorname{Br} 81: 281-288$

12. Siebenrock KA, Schaller $C$, Tannast $M$ et al (2014) Anteverting periacetabular osteotomy for symptomatic acetabular retroversion: results at ten years. J Bone Joint Surg Am 96:1785-1792

13. Siebenrock KA, Schöll E, Lottenbach M et al (1999) Bernese periacetabular osteotomy. Clin Orthop Relat Res (363):9-20

14. Siebenrock KA, Schoeniger R, Ganz R (2003) Anterior femoro-acetabular impingement due to acetabular retroversion. Treatment with periacetabular osteotomy. J Bone Joint Surg Am 85:278-286

15. Siebenrock KA, Steppacher SD, Albers CE et al (2013) Diagnosis and Management of developmental dysplasia of the hip from triradiate closure through young adulthood. J Bone Joint Surg Am 95:748-755

16. Sink EL, Leunig M, Zaltz l et al (2012) Reliability of a complication classification system for orthopaedic surgery. Clin Orthop Relat Res 470:2220-2226

17. Steppacher SD, Lerch TD, Gharanizadeh $\mathrm{K}$ et al (2014) Size and shape of the lunate surface in different types of pincer impingement: theoretical implications for surgical therapy. Osteoarthr Cartil 22:951-958

18. Steppacher SD, Tannast M, Ganz R et al (2008) Mean 20-year followup of Bernese periacetabular osteotomy. Clin Orthop Relat Res 466:1633-1644

19. Steppacher SD, Tannast M, Werlen S et al (2008) Femoral morphology differs between deficient and excessive acetabular coverage. Clin Orthop Relat Res 466:782-790

20. Steppacher SD, Zurmuhle CA, Puls M et al (2015) Periacetabular osteotomy restores the typically excessive range of motion in dysplastic hips with a spherical head. Clin Orthop Relat Res 473:1404-1416

21. Tannast M, Hanke MS, Zheng Getal (2015) What are the radiographic reference values for acetabular under- and overcoverage? Clin Orthop Relat Res 473:1234-1246

22. Tannast M, Pfannebecker P, Schwab JM et al (2012) Pelvic morphology differs in rotation and obliquity between developmental dysplasia of the hip and retroversion. Clin Orthop Relat Res 470:3297-3305

23. Tannast M, Siebenrock KA, Anderson SE (2007) Femoroacetabular impingement: radiographic diagnosis - what the radiologist should know. Am J Roentgenol 188:1540-1552

24. Thawrani D, Sucato DJ, Podeszwa DA et al (2010) Complications associated with the Bernese periacetabular osteotomy for hip dysplasia in adolescents. J Bone Joint Surg Am 92:1707-1714

25. Troelsen A, Elmengaard B, Soballe K (2009) Medium-term outcome of periacetabular osteotomy and predictors of conversion to total hip replacement. J Bone Joint Surg Am 91:2169-2179

26. Trumble SJ, Mayo KA, Mast JW (1999) The periacetabular osteotomy. Minimum 2 year followup in more than 100 hips. Clin Orthop Relat Res 363:54-63

27. Calvert PT, August AC, Albert JS et al (1987) The Chiari pelvic osteotomy. A review of the long-term results. J Bone Joint Surg Br 69:551-555
28. Clohisy JC, Barrett SE, Gordon JE et al (2005) Periacetabular osteotomy for the treatment of severe acetabular dysplasia. J Bone Joint Surg Am 87:254-259. doi:10.2106/JBJS.D.02093

29. Dahl LB, Dengsø K, Bang-Christiansen K, Petersen MM, Stürup J (2014) Clinical and radiological outcome after periacetabular osteotomy: A cross sectional study of 127 hips operated on from 1999-2008. Hip Int 24(4):369-380 (Epub 2014 Apr 8) doi:10.5301/hipint.5000129

30. Grammatopoulos G, Wales J, Kothari A, Gill HS, Wainwright A, Theologis T (2016) What is the early/mid-term survivorship and functional outcome after Bernese periacetabular osteotomy in a pediatric surgeon practice? Clin Orthop Relat Res 474(5):1216-1223. doi:10.1007/s11999-0154386-7

31. Guille JT, Forlin E, Kumar SJ, MacEwen GD (1992) Triple osteotomy of the innominate bone in treatment of developmental dysplasia of the hip. JPediatr Orthop 12:718-721

32. Hartig-Andreasen C, Troelsen A, Thillemann TM, Søballe K (2012) What factors predict failure 4 to 12 years after periacetabular osteotomy? Clin Orthop Relat Res 470:2978-2987. doi:10.1007/s11999012-2386-4

33. Hellemondt GG van, Sonneveld $H$, Schreuder MHE et al (2005) Triple osteotomy of the pelvis for acetabular dysplasia: results at a mean followup of 15 years. J Bone Joint Surg Br 87:911-915. doi:10.1302/0301-620X.87B7.15307

34. Hasegawa $Y$, Iwase T, Kitamura S et al (2002) Eccentric rotational acetabular osteotomy for acetabular dysplasia: follow-up of one hundred and thirty-two hips forfive to ten years. JBone Joint Surg Am 84-A:404-410

35. Kleuver M de, Kooijman MA, Pavlov PW, Veth RP (1997) Triple osteotomy of the pelvis for acetabular dysplasia: results at 8 to 15 years. J Bone Joint Surg $\operatorname{Br} 79(2): 225-229$

36. Lack W, Windhager R, Kutschera HP, Engel A (1991) Chiari pelvic osteotomy for osteoarthritis secondary to hip dysplasia. Indications and longterm results. JBone Joint Surg Br 73:229-234

37. Nakamura S, Ninomiya S, Takatori Y et al (1998) Long-term outcome of rotational acetabular osteotomy: 145 hips followed for $10-23$ years. Acta Orthop Scand 69:259-265

38. Nozawa M, Shitoto K, Matsuda K et al (2002) Rotational acetabular osteotomy for acetabular dysplasia. A follow-up for more than ten years. J Bone Joint Surg Br 84:59-65

39. Ohashi H, Hirohashi K, Yamano Y (2000) Factors influencing the outcome of Chiari pelvic osteotomy: a long-term follow-up. J Bone Joint Surg $\mathrm{Br}$ 82:517-525

40. Pedersen AB, Mehnert F, Havelin LI, Furnes O, Herberts P, Kärrholm J, Garellick G, Mäkela K, Eskelinen A, OvergaardS(2014)Association between fixation technique and revision risk in total hip arthroplasty patients younger than 55 years of age. Results from the Nordic Arthroplasty Register Association. Osteoarthr Cartil 22(5):659-667 (Epub 2014 Mar 13) doi:10.1016/j.joca.2014.03.005

41. Peters CL, Erickson JA, Hines JL (2006) Early results of the Bernese periacetabular osteotomy: the learning curve at an academic medical center. J Bone Joint Surg Am 88:1920-1926. doi:10.2106/ JBJS.E.00515

42. Siebenrock KA, Schöll E, Lottenbach M, Ganz R (1999) Bernese periacetabular osteotomy. Clin Orthop Relat Res 363:9-20

43. Steppacher SD, Tannast M, Ganz R, Siebenrock KA (2008) Mean 20-year followup of Bernese periacetabular osteotomy. Clin Orthop Relat Res 466:1633-1644. doi:10.1007/s11999-008-0242-3

44. Takatori $Y$, Ninomiya S, Nakamura S et al (2001) Long-term results of rotational acetabular osteotomy in patients with slight narrowing of the joint space on preoperative radiographic findings. J Orthop Sci 6:137-140. doi:10.1007/s0077610060137

45. Trumble SJ, Mayo KA, Mast JW (1999) The periacetabular osteotomy. Minimum 2 year followup in more than 100 hips. Clin Orthop Relat Res 363:54-63

46. Troelsen A, Elmengaard B, Søballe K (2009) Medium-term outcome of periacetabular osteotomy and predictors of conversion to total hip replacement. J Bone Joint Surg Am 91:2169-2179. doi:10.2106/JBJS.H.00994

47. Yanagimoto $S$, Hotta H, Izumida R, Sakamaki $T$ (2005) Long-term results of Chiari pelvic osteotomy in patients with developmental dysplasia of the hip: Indications for Chiari pelvic osteotomy according to disease stage and femoral head shape. J Orthop Sci 10:557-563. doi:10.1007/s00776-005-0942-4

48. Yasunaga $Y$, Ochi M, Shimogaki K et al (2004) Rotational acetabular osteotomy for hip dysplasia: 61 hips followed for 8-15 years. Acta Orthop Scand 75:10-15. doi:10.1080/00016470410001708020 